\title{
Rush sublingual immunotherapy in canine atopic dermatitis: a prospective pilot study
}

\author{
M. Fujimura, H. Ishimaru \\ Fujimura Animal Allergy Hospital 10-26 5-choume Aomatanihigashi, \\ Mino, Osaka, 562-0022, Japan
}

\begin{abstract}
Twenty dogs with canine atopic dermatitis (CAD) were treated with rush sublingual immunotherapy (SLIT), with a 48 hour build-up phase and 6 months maintenance phase (treated by antigen once every 3-4 weeks). The canine atopic dermatitis extent and severity index (CADESI)-4 was evaluated before treatment (baseline) and after 6 months. An open, non-controlled, non-randomized pilot trial was conducted to assess the effectiveness and safety of rush SLIT for environmental allergen extracts (Dematophagoides pteronyssinus and D.farinae mix and other). Three dogs dropped out and 17 dogs finished the trial. CADESI-4 at baseline was 60.6 \pm 27.1 (range 17-107, $\mathrm{n}=17$ ). After 6 months of SLIT treatment, CADESI-4 was 37.4 \pm 36.0 (range 5-152, $n=17)(\mathrm{p}<0.01$ ), which was a $38.3 \%$ reduction. A significant improvement, defined as a CADESI-4 reduction of $>30 \%$, was observed in 13 out of 17 dogs (76\%). A moderate improvement, defined as a CADESI-4 reduction of $\leqq 30 \%$, was observed in 2 dogs $(12 \%)$. In the other 2 dogs (12\%), CADESI-4 worsened or showed no change. However, no severe adverse effects were observed during the trial.

Therefore, rush SLIT against environmental allergen extract for CAD showed effectiveness and safety as evidenced by the reduction of CADESI-4 after 6 months SLIT without severe adverse effects.
\end{abstract}

Key words: atopic dermatitis, dog, sublingual immunotherapy, rush

\section{Introduction}

In sublingual immunotherapy (SLIT), specific allergen extracts are delivered into the oral cavity, as opposed to subcutaneous immunotherapy (SCIT) (Bousquet PJ et al. 2009, Radulovic S et al. 2011). This treatment is widely used in Europe to treat allergic respiratory disease and atopic dermatitis in humans (Cadario G et al. 2007, Bousquet PJ et al. 2009). Furthermore, this treatment is being used to treat CAD in the United States (Olivry T et al. 2015, DeBoer DJ et al. 2016). Compared to SCIT, SLIT is considered a more convenient and safer approach for the treatment of allergies (Bousquet PJ et al. 2009, Radulovic S et al. 2011).

One of the disadvantages of SCIT is that it requires a long period of build-up phase and maintenance phase. There are some rush protocols to shorten the period of build-up phase and move into maintenance phase faster than the current standard protocol (Mueller SR et al. 2001, Trimmer AM et al. 2005).

To improve the current standard protocol, we tested the effectiveness and safety of rush SLIT against 
environmental allergen extract for CAD. CADESI-4 was used to measure effectiveness.

\section{Materials and Methods}

\section{Animals}

All dogs were diagnosed with canine atopic dermatitis at one clinic (Fujimura Animal Allergy Hospital, Osaka, Japan). The study started in January 2014 and concluded in June 2015 (Table 1).
(Favrot $\mathrm{C}$ et al. 2010). To determine the identity of the sensitized antigens an intradermal allergy test was performed for 24 selected antigens. These antigens were subdivided into six environmental antigen groups (mite mix; Dermatophagoides farina and Dermatophagoides pteronyssinus, dust, epithelia, tree, weed, grass, mold) and flea antigen. The majority of commercial allergen preparations were purchased from Greer Laboratories (Lenoir, USA). The remainder (Japanese cedar) was obtained from Torii Medicine (Tokyo, Japan). The mixed house dust mite extract was used at a concentration of $1,000 \mathrm{PNU} / \mathrm{ml}$

Table 1. Rush SLIT with $48 \mathrm{~h}$ build up phase and 6 months maintenance phase.

\begin{tabular}{|c|c|c|c|c|c|}
\hline \multirow{2}{*}{ No. } & \multirow{2}{*}{ Breed } & \multirow{2}{*}{ Antigen } & \multirow{2}{*}{ Adverse Effects } & \multicolumn{2}{|c|}{ CADESI-4 } \\
\hline & & & & PRE & POST \\
\hline 1 & French Bulldog & HDM & Vomiting & 77 & 54 \\
\hline 2 & Miniature Dachshund & HDM & None & 68 & 38 \\
\hline 3 & Cavalier King Charles Spaniel & HDM & None & 39 & 12 \\
\hline 4 & German Shepherd Dog & HDM & Itch & 19 & ND \\
\hline 5 & Golden Retriever & HDM & Itch & 83 & 152 \\
\hline 6 & Toy Poodle Mix & HDM & None & 17 & 3 \\
\hline 7 & French bulldog & HDM, 7Grass mix and Velvet & Itch & 99 & 740 \\
\hline 8 & Shiba Inu & HDM and Cotton & None & 75 & 46 \\
\hline 9 & Shi Tsu & Mold & Itch & 48 & 24 \\
\hline 10 & German Shepherd Dog & HDM & None & 35 & 20 \\
\hline 11 & Shiba Inu & 14 Grass mix & None & 48 & 11 \\
\hline 12 & Toy Poodle & HDM & Itch & 103 & 48 \\
\hline 13 & Papillon & HDM & None & 25 & ND \\
\hline 14 & Shiba Inu & HDM and 7 Grass mix & None & 44 & 15 \\
\hline 15 & Miniature Pinscher & HDM & Itch & 31 & ND \\
\hline 16 & Golden Retriever & HDM & Itch & 25 & 27 \\
\hline 17 & Shiba Inu & HDM & Itch (paw) & 71 & 5 \\
\hline 18 & Toy Poodle & HDM & Itch & 107 & 61 \\
\hline 19 & Chihuahua & HDM & Vomiting & 54 & 32 \\
\hline \multirow[t]{2}{*}{20} & Shiba Inu & 7 Grass mix, Velvet and JC & Itch & 38 & 14 \\
\hline & Mean \pm SD & & & $60.6 \pm 27.1$ & $37.4 \pm 36.0$ \\
\hline
\end{tabular}

PRE: Baseline, POST: After 6 months of treatment

ND: Not determined because Dog No. 4, 13 and 15 dropped out from trial due to lack of followup, poor client compliance or early discontinuation for mechanical reasons

JC: Japanese cedar pollen

\section{Diagnosis of CAD and sensitized allergen}

The diagnosis of CAD was made by ruling out other causes of the itch. All dogs received flea control and appropriate treatment for scabies mites. If bacterial pyoderma and yeast (Malassezia dermatitis) was diagnosed by cytology, it was treated mainly by shampoo therapy. All dogs underwent an elimination diet using „hypoallergenic” foods (Hill's prescription diet canine z/d Ultra: Hill's Pet Nutrition, Topeka, KS, USA; or Royal Canin Veterinary Diet Sensitivity Control: Royal Canin, Aimargue, France; or Iams Veterinary Formulas FP: Cincinnati, Ohio, USA) for at least 8 weeks. Diagnosis of CAD was based on compatible history and clinical signs of Favrot's criteria and $200 \mathrm{PNU} / \mathrm{ml}$. House dust extract was used at a concentration of $100 \mathrm{PNU} / \mathrm{ml}$ and other antigens were at a concentration of 1,000 PNU/ml. All extracts were prepared and diluted as sterile diluents. During the intradermal allergy test, dogs were premedicated with atropine sulfate $(0.04 \mathrm{mg} / \mathrm{kg}$, subcutaneously $)$ and sedated with xylazine $(0.15 \mathrm{mg} / \mathrm{kg}$, intravenously).

\section{Canine Atopic Dermatitis Extent and Severity Index (CADESI)-4}

CADESI-4 was used to assess lesion severity (Olivry $\mathrm{T}$ et al. 2014). A severity of erythema, lichenification, excoriations/alopecia was assessed at 20 body 
sites using a scale from $0-3(0=$ none, $1=$ mild, $2=$ moderate, and $3=$ severe). Benchmarks for mild, moderate and severe AD skin lesions are 10, 35 and 60 , respectively. CADESI-4 was evaluated before treatment (baseline) and after 6 months treatment. No other therapies (e.g., oral corticosteroids, cyclosporine, or antimicrobial) were allowed during the 6 months treatment period.

\section{Sublingual rush immunotherapy}

Twenty dogs were treated with rush SLIT with the following antigens.14 dogs were treated only with HDM (Dermatophagoides farina and Dermatophagoides pteronyssinus), 1 dog with HDM, 7 Grass mix (Poa pratensis, Dactylis glomerata, Agrostis alba, Phleum pratense, Anthoxanthum odoratum, Festuca elatior, Lolium perenne) and Velvet extracts, $1 \mathrm{dog}$ with HDM and Cotton extract, 1 dog with Mold extracts (Penicillium notatum), 1 dog with 14 Grass mix extracts (7 Grass mix, Cypress bald, Velvet, Bermuda, Dandelion, Mugwort, Plantain english and Ragweed short), 1 dog with HDM and 7 Grass mix extracts and 1 dog with 7 Grass mix, Velvet and Japanese cedar pollen (Table 1). All antigen extracts were diluted in sterile diluents (containing $0.4 \%$ phenol preservative). No antigens contained $50 \%$ glycerin saline.

Rush sublingual immunotherapy (SLIT) consisted of a 48 hour build-up phase (Table 2) and 6 months maintenance phase (treated with antigen once every 3-4 weeks). Sublingual administration was performed by a veterinarian. Exclusion criteria included treatment with systemic or highly potent systemic corticosteroids or immunosuppressant agents. For concomitant therapies, temporary use of corticosteroid spray or ointment was allowed.

\section{Results}

Out of 20 dogs, 3 dogs (No.4 German Shepherd Dog, No.13 Papillon, and No.15 Miniature Pinscher) dropped out before the end of the 6 months of treatment. The reasons for the drop out were as follows: Dog No.4 showed poor response to SLIT and had to switch to SCIT after 3 months of maintenance phase. Dog No. 13 showed low compliance to the treatment. Dog No. 15 was excluded due to severe itch requiring oral steroid treatment. These 3 dogs were excluded from the data.

CADESI-4 at baseline was $60.6 \pm 27.1$ (range $17-107, \mathrm{n}=17)$. After 6 months of SLIT, CADESI-4 was significantly reduced to $37.4 \pm 36.0$ (range:3-152, $\mathrm{n}=17)(\mathrm{p}<0.01)$. This was a $38.3 \%$ reduction compared to baseline. The dogs were divided into 3 groups as defined by their improvement rate (Cadario et al. 2007). A significant improvement defined as a CADESI-4 reduction of $>30 \%$, was observed in 13 out of 17 dogs $(76 \%)$. A moderate improvement defined as a CADESI-4 reduction of $\leqq 30 \%$, was observed in 2 cases $(12 \%)$. In the last 2 cases (12\%), CADESI-4 worsened or showed no change (Table 1).

No severe adverse effects were observed during the trial. In the build-up phase, weak itch was seen in 10 dogs and vomiting was seen in 2 dogs. There was moderate itch on the paws which disappeared in a short time (Table 1). In the maintenance phase, vomiting was seen in $1 \mathrm{dog}$ (data not shown). None of the dogs had to stop the trial due to adverse effects. However, Dog Nos. 2, 5 and 9 received steroid ointment or spray once a week.

Table 2. Sublingual rush immunotherapy protocol 48 hour build-up phase.

\begin{tabular}{|c|c|c|c|}
\hline & \multicolumn{3}{|c|}{ Antigen concentration } \\
\hline & $200 \mathrm{PNU} / \mathrm{mL}$ & $2,000 \mathrm{PNU} / \mathrm{mL}$ & $20,000 \mathrm{PNU} / \mathrm{mL}$ \\
\hline Day 1 (9:00) & $0.05 \mathrm{~mL}$ & & \\
\hline Day $1(10: 00)$ & $0.1 \mathrm{~mL}$ & & \\
\hline Day 1 (11:00) & $0.2 \mathrm{~mL}$ & & \\
\hline Day $1(12: 00)$ & $0.4 \mathrm{~mL}$ & & \\
\hline Day 1 (13:00) & & $0.05 \mathrm{~mL}$ & \\
\hline Day $1(14: 00)$ & & $0.1 \mathrm{~mL}$ & \\
\hline Day $1(15: 00)$ & & $0.2 \mathrm{~mL}$ & \\
\hline Day $1(16: 00)$ & & $0.4 \mathrm{~mL}$ & \\
\hline Day $2(9: 00)$ & & & $0.05 \mathrm{~mL}$ \\
\hline Day $2(10: 00)$ & & & $0.1 \mathrm{~mL}$ \\
\hline Day $2(11: 00)$ & & & $0.2 \mathrm{~mL}$ \\
\hline
\end{tabular}

PNU: protein nitrogen units

Maintenance phase: SLIT administration threshold concentration on day 2, thereafter each 3-4 weeks. The threshold concentration varied according to dog. Most threshold concentration was 20,000 PNU/mL and administration volume was $0.2 \mathrm{~mL}$ (4,000 PNU). 


\section{Statistical Analysis}

Statistical analysis was performed using a Wilcoxon signed-rank test, and statistical significance was defined as $\mathrm{p}<0.01$.

\section{Discussion}

In this trial, rush SLIT showed effectiveness for the treatment of CAD. Administration frequency of maintenance phase was once every 3-4 weeks. This administration frequency was based on the maintenance phase of SCIT (Olivry T et al. 2015). In human study, administration frequency of the maintenance phase is once per day (Cadario G, et al. 2007), but, as for our method, an effectiveness for dogs were clear.

There was itch and vomiting in the 48 hour build-up phase and vomiting in the maintenance phase. However, these were not severe. This study shows that rush SLIT is safe. The mild adverse effects can readily be treat dog by owner at home. However, further investigation using a greater sample size may be needed to confirm these results.

Eighty-eight percent of the dogs showed improvement, which was higher than that of a previous trial of $60 \%$ (Olivry $\mathrm{T}$ et al. 2015). The major difference of two trials is who administered the treatment. In our trial, a trained veterinarian treated the dogs. In contrast, dog owners administered the treatment in the previous trial. This is probably the reason for the difference in improvement rate since a trained professional can more easily handle the dogs.

Dog No. 4 (G. Shepherd) dropped out before end of the trial, because SLIT was not effective and SCIT had to be used. In this case, the dog showed drivel and it appeared that the G. Shepherd was not suitable for SLIT. Moreover, 3 dogs which did not show improvement after 6 months SLIT, did improve with SCIT. But changing from SLIT to SCIT raises concern about adverse effects. Anaphylactic shock was observed in one dog who switched from SLIT to SCIT (data not shown). Also in a previous trial, in dogs which showed no response after SCIT, $49 \%$ of them showed improvement after changing to SLIT. These results indicate that, in non-responsive cases, changing from SLIT to SCIT or vice versa might be beneficial.

\section{Conclusion}

Rush SLIT is effective and safe for CAD sensitized to environmental allergens. Future research will be needed to compare side-by-side the effectiveness of rush SLIT to SCIT with a large sample size.

\section{Acknowledgments}

The author thanks Dr Ester M Yoo for translations and revising the manuscript.

\section{References}

Canonica GW, Bousquet J, Casale T, Lockey RF, Baena-Cagnani CE, Pawankar R, Potter PC, Bousquet PJ, Cox LS, Durham SR, Nelson HS, Passalacqua G, Ryan DP, Brozek JL, Compalati E, Dahl R, Delgado L, van Wijk RG, Gower RG, Ledford DK, Filho NR, Valovirta EJ, Yusuf OM, Zuberbier T, Akhanda W, Almarales RC, Ansotegui I, Bonifazi F, Ceuppens J, Chivato T, Dimova D, Dumitrascu D, Fontana L, Katelaris $\mathrm{CH}$, Kaulsay R, Kuna P, Larenas-Linnemann D, Manoussakis M, Nekam K, Nunes C, O'Hehir R, Olaguibel JM, Onder NB, Park JW, Priftanji A, Puy R, Sarmiento L, Scadding G, Schmid-Grendelmeier P, Seberova E, Sepiashvili R, Solé D, Togias A, Tomino C, Toskala E, Van Beever H, Vieths S (2009) Sub-lingual immunotherapy: World Allergy Organization Position Paper 2009. Allergy 64: 1-59.

Cadario G, Galluccio AG, Pezza M, Appino A, Milani M, Pecora S, Mastrandrea F (2007) Sublingual immunotherapy efficacy in patients with atopic dermatitis and house dust mite sensitivity: a prospective study. Curr Med Res Opin 23: 2503-2506.

DeBoer DJ, Verbrugge M, Morris M (2016) Clinical and immunological responses of dust mite sensitive, atopic dogs to treatment with sublingual immunotherapy (SLIT). Vet Dermatol 8. doi: 10.1111/vde.12284.

Favrot C, Steffan J, Seewald W, Picco F (2010) A prospective study on the clinical features of chronic canine atopic dermatitis and its diagnosis. Vet Dermatol 21: 23-31.

Mueller SR, Bettenay SV (2001) Evaluation of safety of an abbreviated course of injections of allergen extracts (rush immunotherapy) for the treatment of dogs with atopic dermatitis. Am J Vet Res 62: 307-310.

Olivry T, Saridomichelakis M, Nuttall T, Bensignor E, Griffin CE, Hill PB: International Committee on Allergic Diseases of Animals (ICADA). (2014) Validation of the Canine Atopic Dermatitis Extent and Severity Index (CADESI)-4, a simplified severity scale for assessing skin lesions of atopic dermatitis in dogs. Vet Dermatol 25: 77-85.

Olivry T, DeBoer DJ, Favrot C, Jackson HA, Mueller RS, Nuttall T, Prelaud P (2015) International Committee on Allergic Diseases of Animals. Treatment of canine atopic dermatitis: 2015 updated guidelines from the International Committee on Allergic Diseases of Animals (ICADA). BMC Vet Res 11: 210.

Radulovic S, Wilson D, Calderon M, Durham S (2011) Systemic reviews of sublingual immunotherapy (SLIT). Allergy 66: 740-752.

Trimmer AM, Griffin CE, Boord MJ, Rosenkrantz WS (2005) Rush allergen specific immunotherapy protocol in feline atopic dermatitis: a pilot study of four cats. Vet Dermatol 16: 324-329 